Scientific Journal of Hamadan Nursing \& Midwifery Faculty - ISSN 2008-2819

\title{
The Relationship Between Organizational Culture and Knowledge Management in Educational-Therapeutic Hospitals \\ (Case study Hamadan Educational-Therapeutic Hospitals in 2017)
}

\author{
Akbar Bahmani' ${ }^{1}$ Amin Biglarkhani ${ }^{* 2}$, Gholam Hossein Fallahinia ${ }^{3}$, Fatemeh Shirani ${ }^{4}$
}

1. PhD, Department of Management, Payame Noor University, Tehran, Iran

2. PhD Student in Information Technology Management, Department of Information Technology Management, Hamadan University of Medical Sciences, Hamadan, Iran

3. Chronic Diseases (Home Care) Research Center, Hamadan University of Medical Sciences, Hamadan, Iran

4. MSc in Nursing, School of Nursing and Midwifery, Hamadan University of Medical Sciences, Hamadan, Iran

\begin{tabular}{ll}
\hline \multicolumn{1}{c}{ Article Info } \\
\hline & \\
Received: & $2017 / 07 / 25$ \\
Accepted: & $2017 / 12 / 18$ \\
Published Online & $2018 / 02 / 6$
\end{tabular}

DOI:

10.30699/sjhnmf.26.3.203

Original Article

Use your device to scan and read the article online

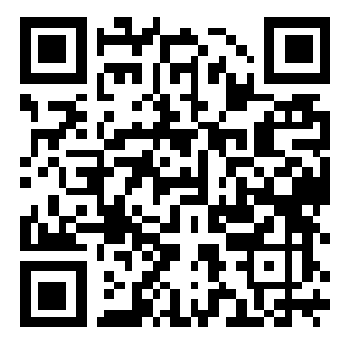

\section{Abstract}

Introduction: Knowledge management in health care system can reduce medical errors, increase the quality of care, organizational learning and promote cooperation, innovation and cause cost reduction. The success of successful organizations lies in the organizational culture and employees' beliefs. The aim of this research was to investigate the relationship between organizational culture and knowledge management in Hamadan educational-therapeutic hospitals.

Methods: The present study was a correlational study-related survey which was carried out in a cross-sectional way on 300 nurses in Hamadan's educational-therapeutic hospitals in 2017. Robbins organizational culture questionnaires and Nanoka Knowledge Management have been used to collect information. Data were analyzed using SPSS24 and descriptive (mean, deviation and variance) and analytical (Pearson correlation coefficient) methods were used.

Results: In this study, the organizational culture of nurses (with a score of 2.5) and knowledge management (with score of 3.16) were in appropriate conditions. There was a positive and significant relationship between organizational culture and knowledge management $(\mathrm{r}=0.639, P=0.001)$. All components of organizational culture with knowledge management have a positive and significant correlation.

Conclusion: According to the results of the study, if organizational culture and its components are improved, the progress and improvement of knowledge management will increase the quality of health care services. It is suggested that in the process of establishing knowledge management in medical centers, attention should be paid to the structural and cultural dimensions of the organization, in particular the development of individual creativity and leadership style, coherence and integrity.

Keywords: Organizational culture, Knowledge management, Hospital, Nurse

\footnotetext{
Corresponding Information

Amin Biglarkhani, PhD Student in Information Technology Management, Department of Information Technology Management, Hamadan University of Medical Sciences, Hamadan, IranE-mail: biglarkhani@gmail.com

Copyright (C) 2018, Sci J Hamadan Nurs Midwifery Fac. This is an open-access article distributed under the terms of the Creative Commons Attribution-noncommercial 4.0 International License which permits copy and redistribute the material just in noncommercial usages, provided the original work is properly cited. How to Cite This Article:

Bahmani A, Biglarkhani A, Fallahinia G H, Shirani F. The Relationship Between Organizational Culture and Knowledge Management in Educational-Therapeutic Hospitals(Case study Hamadan Educational-Therapeutic Hospitals in 2017). Sci J Hamadan Nurs Midwifery Fac. 2018; 26 (3):203-210
} 
اثربخشى خدمات مواجه كردهاند. اين تغييـرات بــهـ افزايش دايش

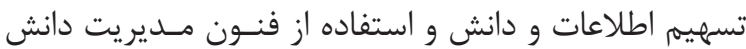

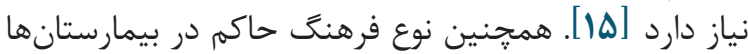

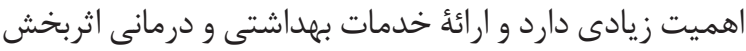

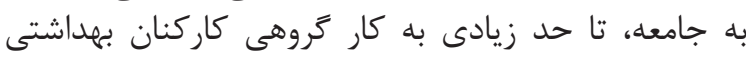

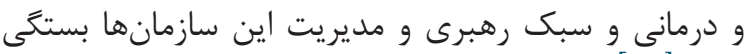

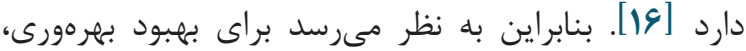

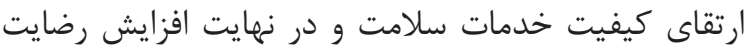

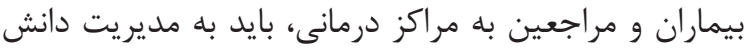

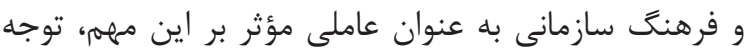

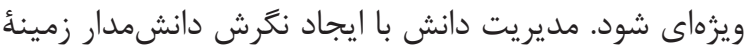

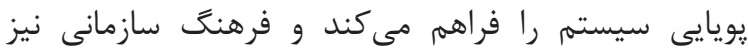

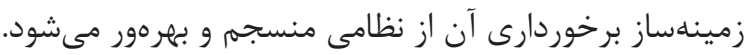

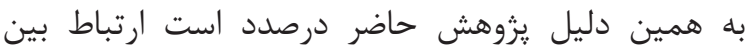

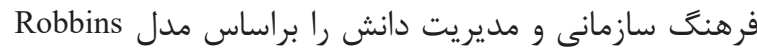

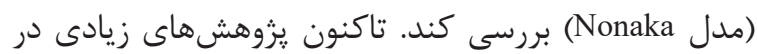

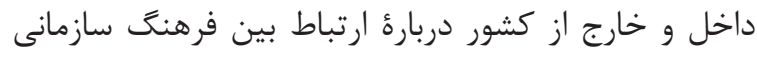

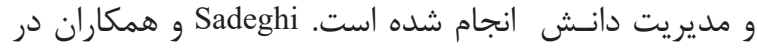

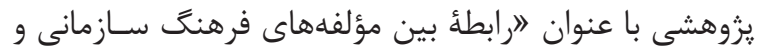

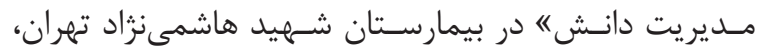

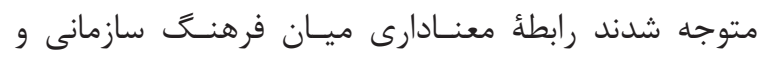

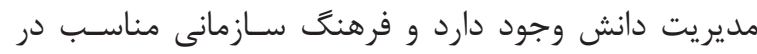

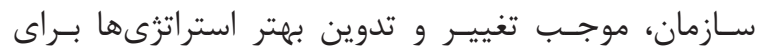

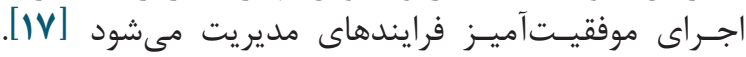
Amiresmaeli

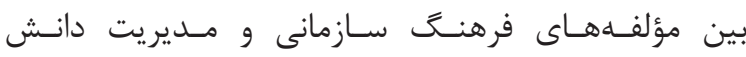

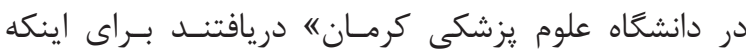

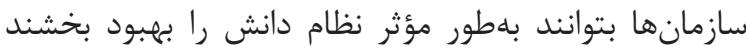

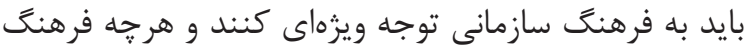

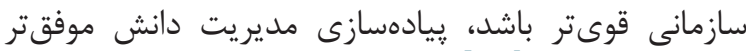

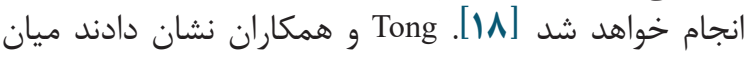

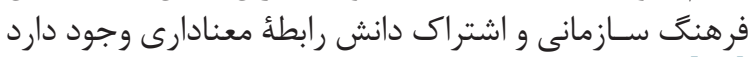

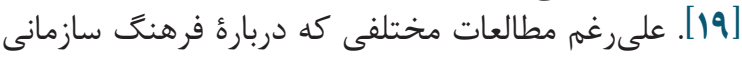

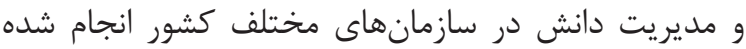

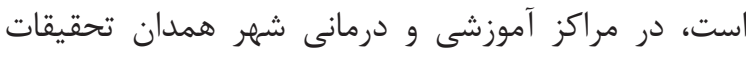

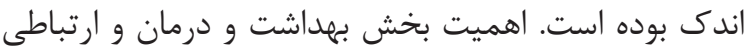

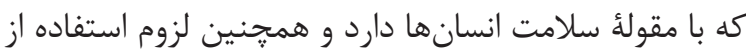

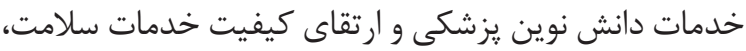
ضرورت انجام اين تحقيق را آشكار مى كند.

\section{روش كار}

يزوهش حاضر از نوع همبستكى است و هدف از انجام

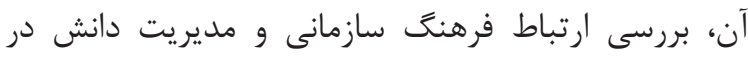

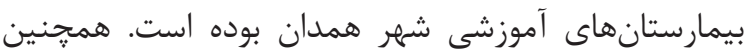

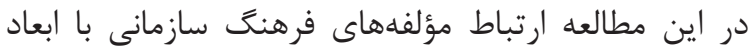

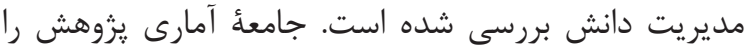

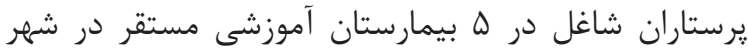

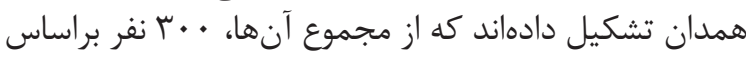

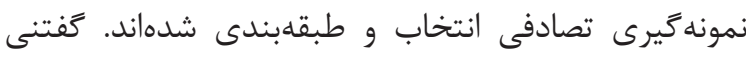
است كه داشتن حداقل يك سال سابقهُ كار و تمايل به شركت

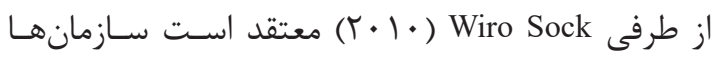

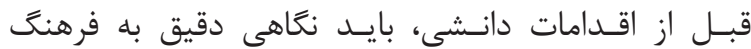

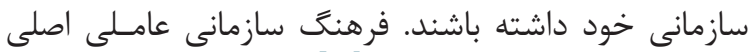

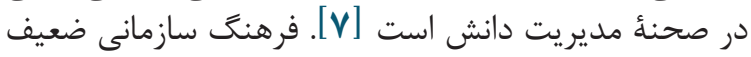

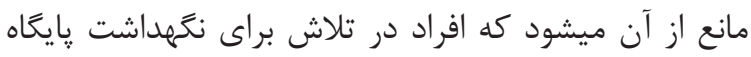

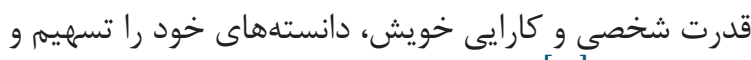

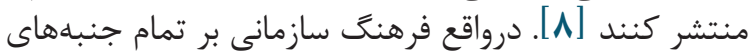

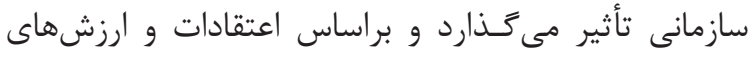

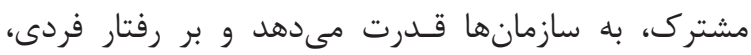

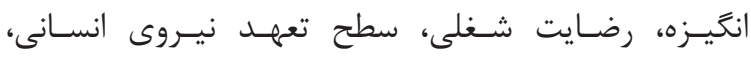

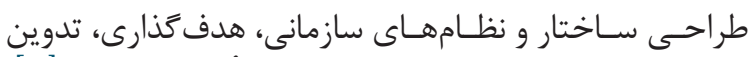

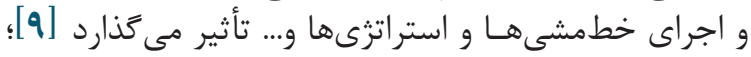

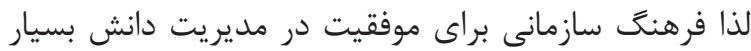

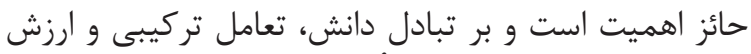

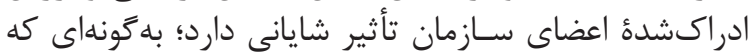

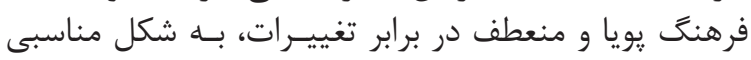

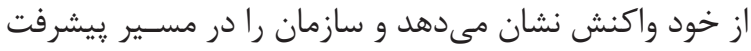

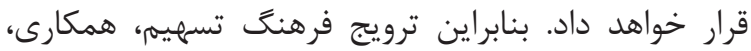

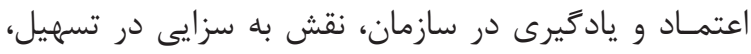

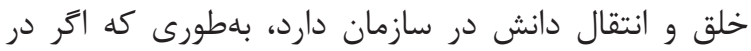

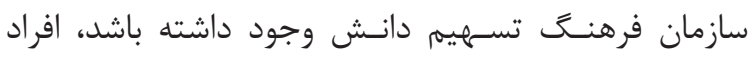

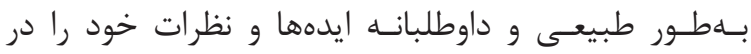

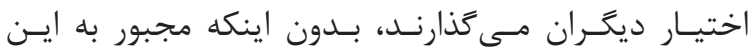

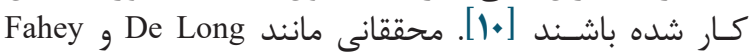

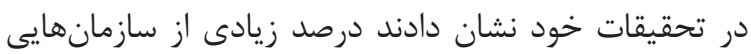

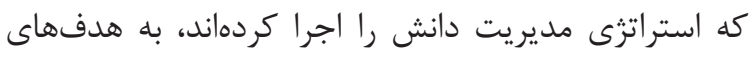

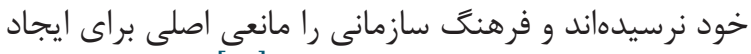

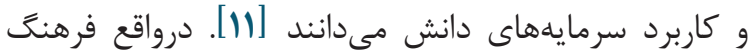

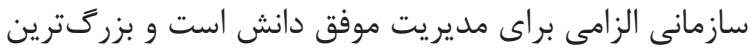

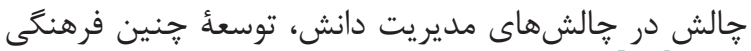

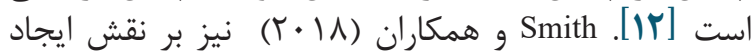

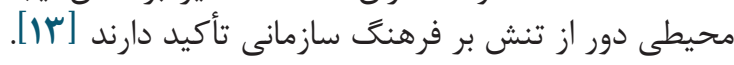

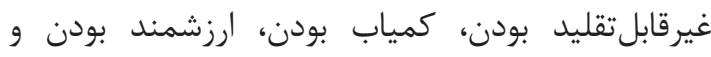

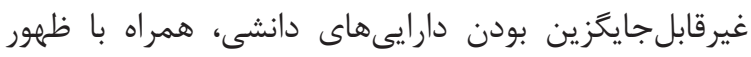

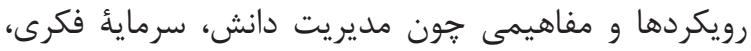

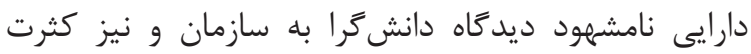

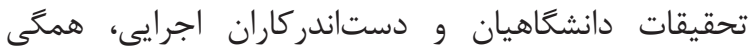

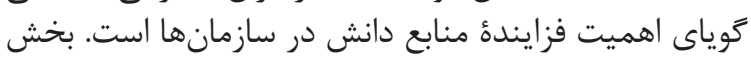

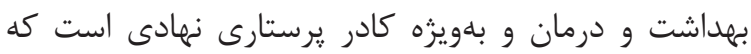

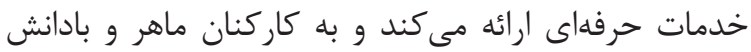

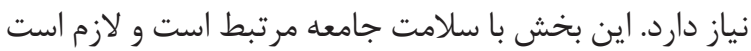

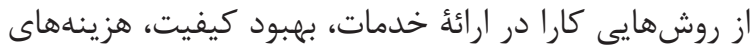

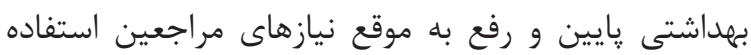

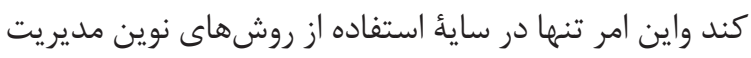

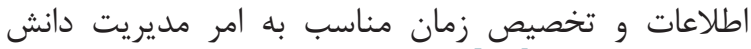

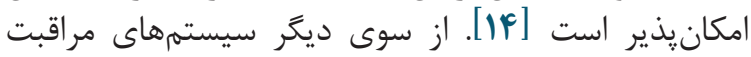

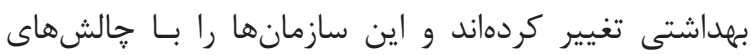

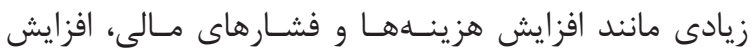
آكاهى مشتريان، رقابتى شدن بازار و ارتقاى كينى كيفيت و هزينه 


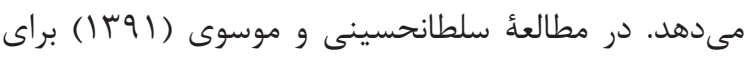

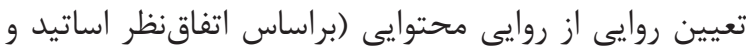

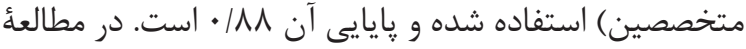

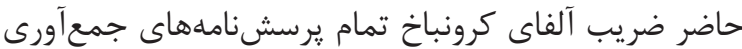

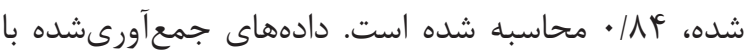

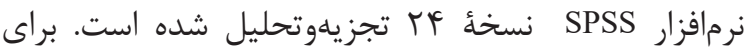

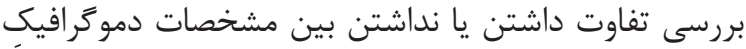

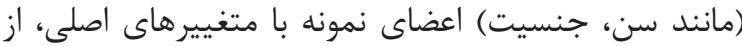

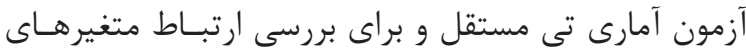

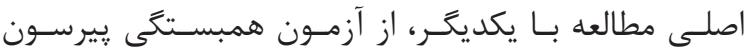

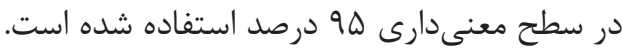

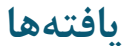

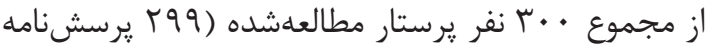

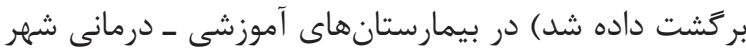

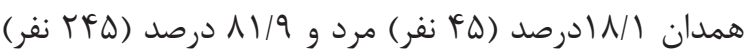

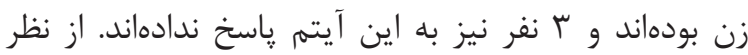

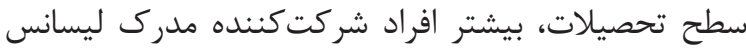

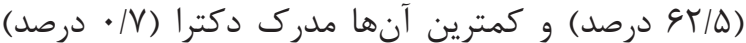

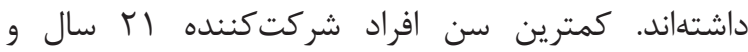

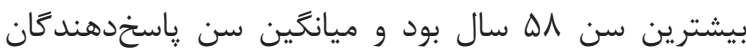

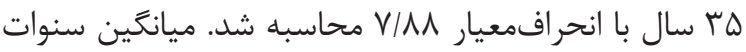

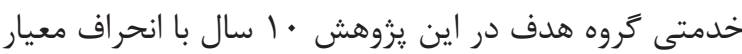

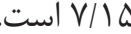

نتايج جدول شمارئ ا نشان مىدهد بالاترين امتياز در

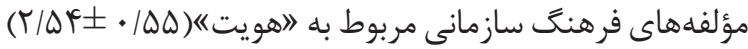

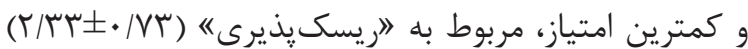

است.

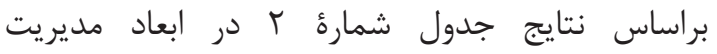

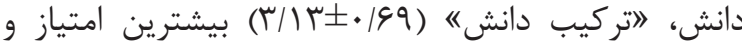

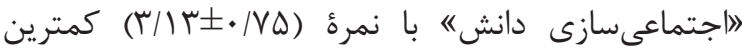

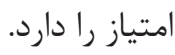

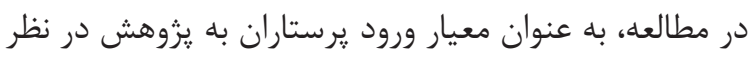

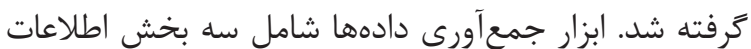

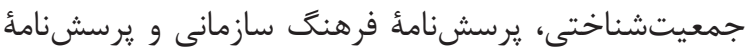

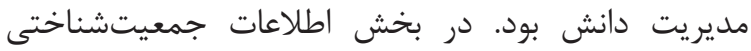

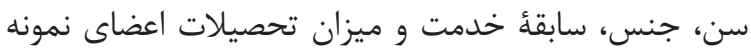

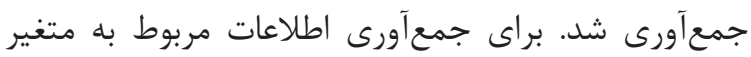

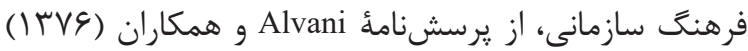

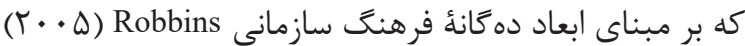

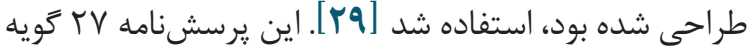

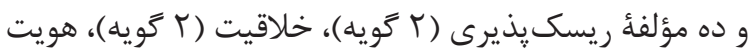

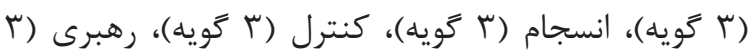

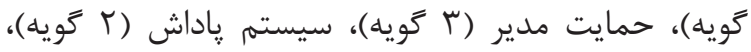

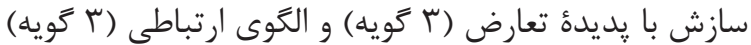

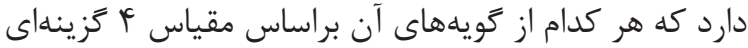

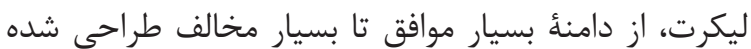

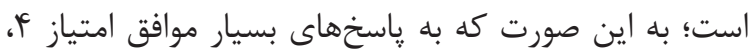

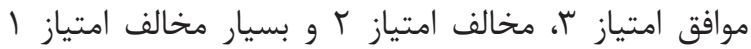

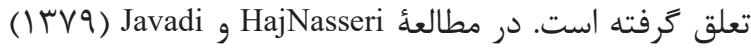

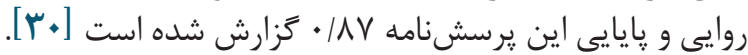

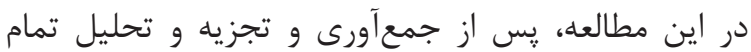

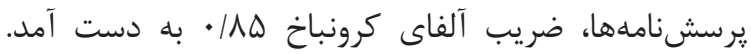

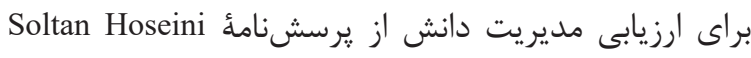

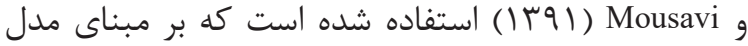
Nonaka

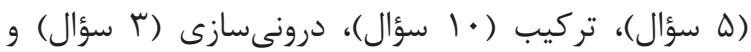

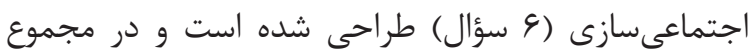

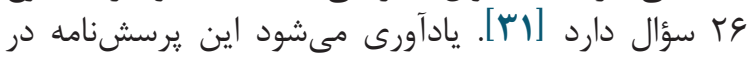

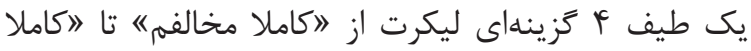

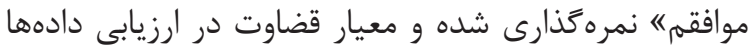

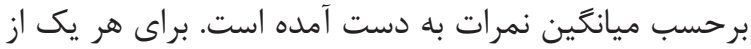

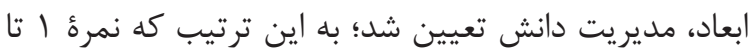

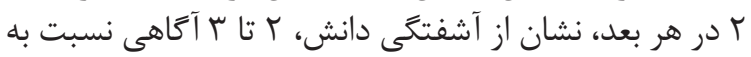

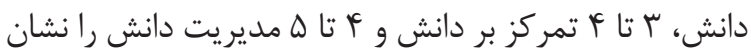

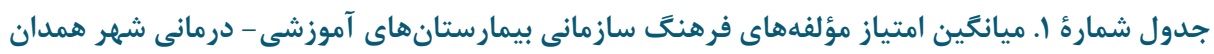

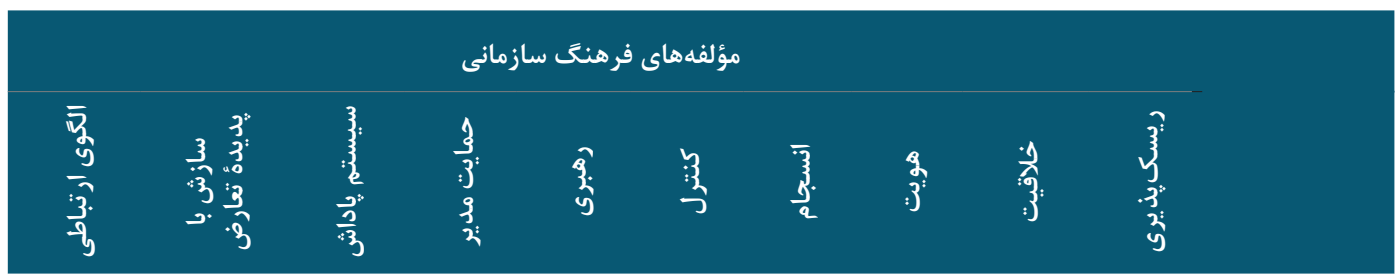

\begin{tabular}{|c|c|c|c|c|c|c|c|c|c|c|}
\hline$r / \varphi$. & $T / Y \Lambda$ & T/ET & $r / \Delta \cdot$ & $r / \Delta V$ & T/QT & $r / \Delta T$ & $T / \Delta F$ & T/FY & Tr/T & ميانخين \\
\hline .195 & $\cdot / \Delta \varphi$ & 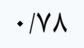 & $\cdot 109$ & $\cdot / D F$ & $\cdot / \Delta \Delta$ &.$/ 9 T$ & $\cdot / \Delta \Delta$ & $\cdot 19 \Delta$ & $\cdot / V r$ & انحرافمعيار \\
\hline$\cdot / 4 q$ & $\cdot / \pi 1$ & $\cdot|9|$ & $\cdot / \pi \Delta$ & $\cdot / \pi q$ & $\cdot / r \cdot$ & $\cdot / 4 q$ & $\cdot|r|$ & . A & $\cdot / D F$ & واريانس \\
\hline $1 / \cdots$ & $1 / \cdot$ & $1 / \cdot$ & $1 / \cdot$ & $1 / \cdot$ & $1 / \cdot$ & $1 / \cdot$ & $1 / \cdot$ & $1 / \cdots$ & $1 / \cdot$ & حداقل \\
\hline |. & $f / \cdot$ & $r /$. & $\boldsymbol{r} / .$. & $r /$. & $r /$. & $f /$. & $r /$. & $r /$. & $r / \cdot$ & حداكثر \\
\hline
\end{tabular}




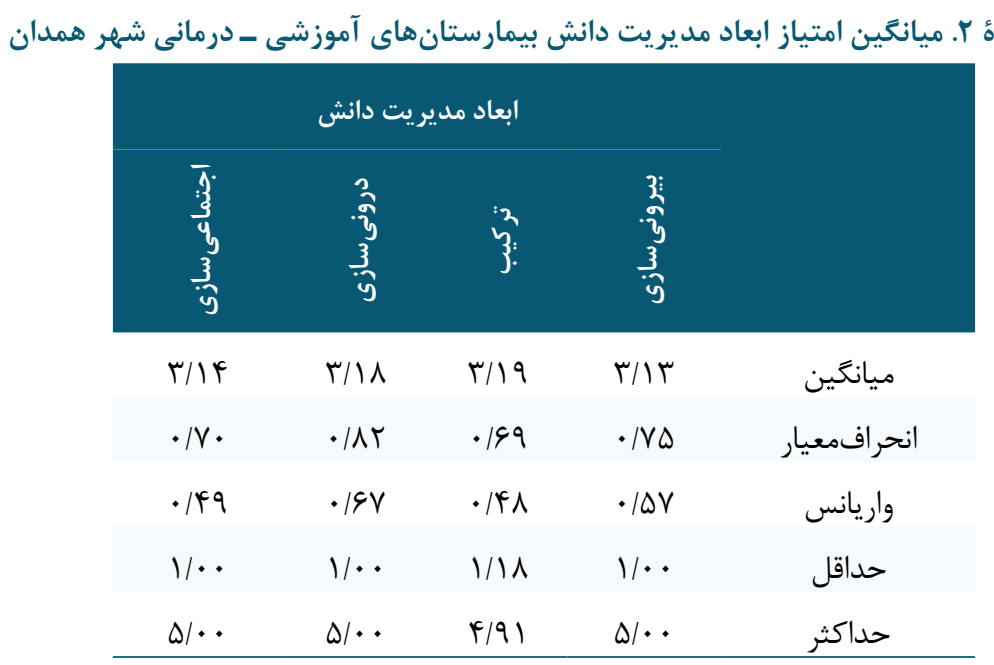

دارد. تمامى مؤلفههاى فرهنَ سازمانى (ريسكيذيرى،

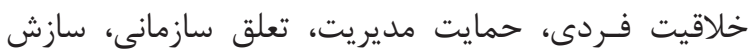

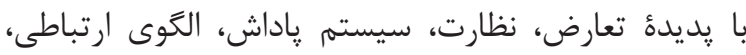

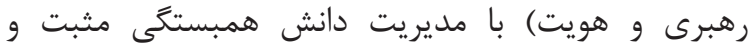

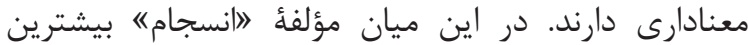

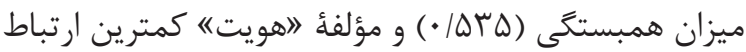

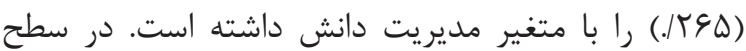

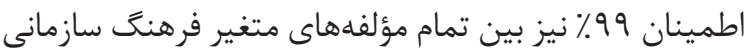
و ابعاد مديرت دانش، همبستخى مثبت و و معنادارى دين ديده مى شود.
بالاترين امتياز فرهنَ سازمانى به بيمارستان قبان قلب

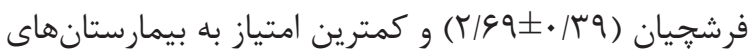

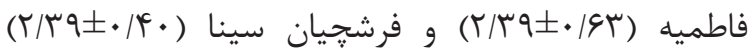

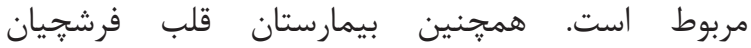

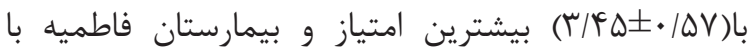
(T/Al|•/QT) هتايج جدول شماره ب نشان مى ندهد در سطح اطمينان

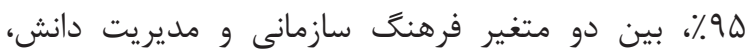

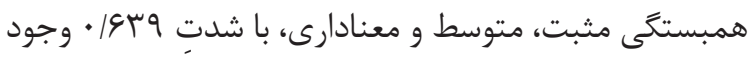

جدول شمارهُ ب. ضريب همبستكى ميان فرهنَ سازمانى و مؤلفههاى آن با مديريت دانش و ابعادش

\begin{tabular}{|c|c|c|c|c|c|c|c|c|c|c|c|}
\hline & ريسك يذيرى & خلاقيت & هويت & انسجام & كنترل & رهبرى & مدمايت & ساداشته & با بديدة & ارتباطى الكَى & فرازمانى \\
\hline بيرونىسازى & $\cdot \mid r \cdot r^{*}$ & 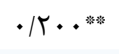 & $\cdot / \mu \ldots * *$ & $\cdot / \mu \cdot V^{* *}$ & - Rer: & $\cdot / T \Delta Q^{* * *}$ & $\cdot / K F \cdot * *$ & 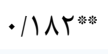 & $\cdot / 19 V^{* *}$ & $\cdot / K r \mu^{0}$ & - \\
\hline تركيب & $\cdot|\Delta F|^{* * *}$ & $\cdot / 4 \& 0^{* * *}$ & . & $\cdot / 499^{* * \%}$ & • & $\cdot \mid r \wedge r^{* * * *}$ & $\cdot|f| 9^{* * m}$ & $\cdot / \mu \ldots$ * *** & $\cdot \pi V Q^{* * *}$ & $\cdot \mid r \mu V^{* * a}$ & - \\
\hline درونىسازى & $\cdot / r V \Lambda^{* m a}$ & $\cdot / Y^{* * t h}$ & $\cdot / 1 \mathrm{rq}^{* * \mathrm{*}}$ & 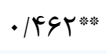 & $\cdot / \Upsilon 4 \Delta^{\text {was }}$ & $\cdot / \mu \cdot \Delta^{* * *}$ & $\cdot / r v Y^{p_{*}^{*}}$ & $\cdot / T V \cdot$ *w & $\cdot \pi V r^{* * *}$ & $\cdot|r \Lambda|^{* * *}$ & - \\
\hline اجتماعىسازى & $\cdot \mid 499^{* * *}$ & $\cdot / 4 \Delta \Delta^{* * *}$ & $\cdot / r \mid Q^{* * *}$ & $\cdot / 4 q 9^{4 * n}$ & $\cdot / r \Delta V^{* * *}$ & $\cdot / \Gamma \xi V^{* * * *}$ & . $/ F \Delta Y^{* * *}$ & . & • & $\cdot|r q|^{* *}$ & - \\
\hline مديريت دانش & •|QU| & $\cdot / \mathcal{C} \Delta \Delta^{*}$ & 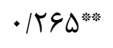 & $\cdot \mid \Delta r \Delta^{* * a}$ & $\cdot / \Gamma 9 V^{* * *}$ & $\cdot / \kappa \cdot \varphi^{k}$ & $\cdot / 4 \Delta Y^{*}$ & & $\cdot / \kappa \cdot \varphi^{c}$ & $. / 4<q^{*}$ & . $\mid \&$ r $9 *$ \\
\hline
\end{tabular}

حيات در آموزش مستمر است و فقط از همين راه مىتوان

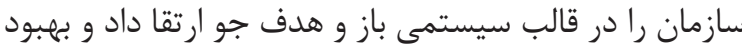

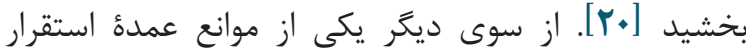

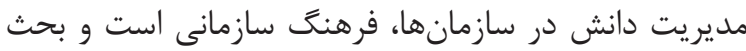

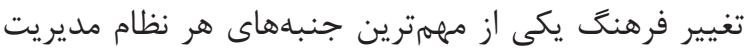

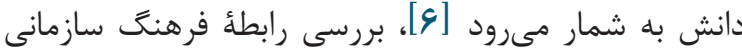

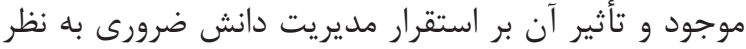

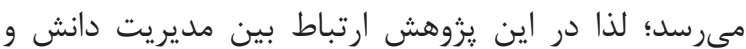

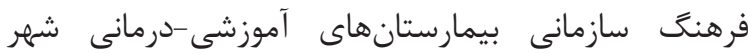

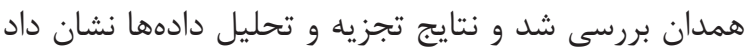

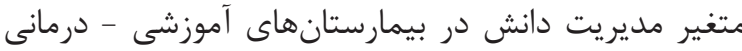
شهر همدان وضعيت مطلوبى دارد، زيرا كسب نمرئ أنسأ ميانگين

دوره צY شماره r - مرداد و شهريور
همانطور كه از نتايج جدول بالا معلوم است، بيشترين

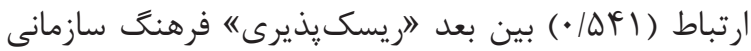

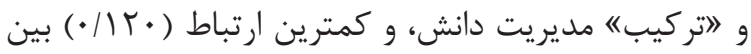

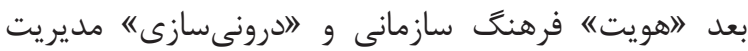
دانش ديده مىشود.

\section{بحث}

بيمارستانها علاوه بر هدف اصلى خود كه ارتقاى سلامت

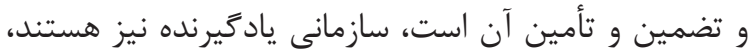

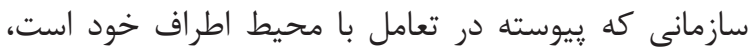

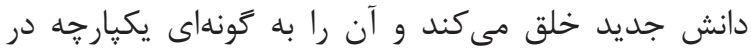

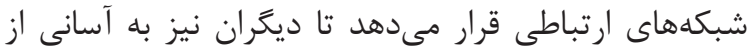

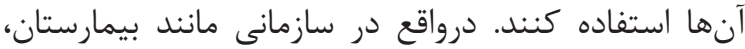




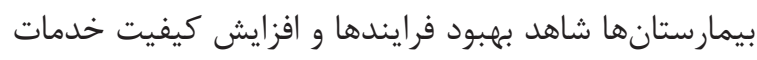

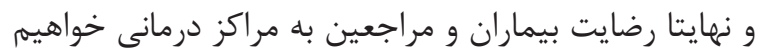
بود.

\section{نتيجه گَيرى}

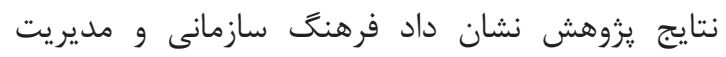
دانش در بيمارستانهاى آموزشى ـ درمانى شانى شهر همدان

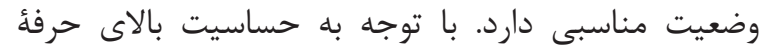

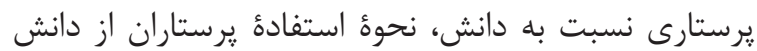

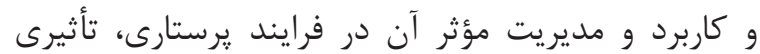

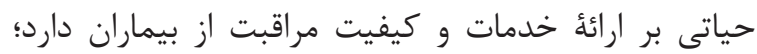

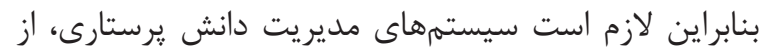

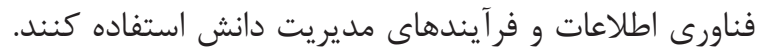

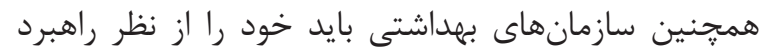

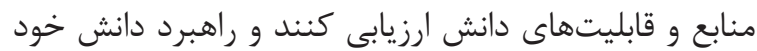

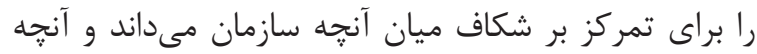

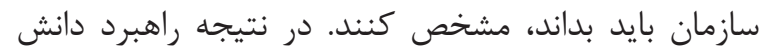

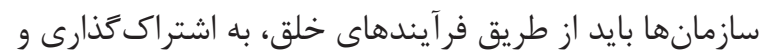

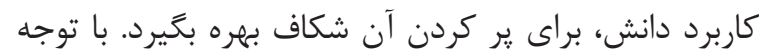

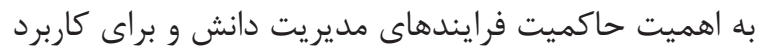

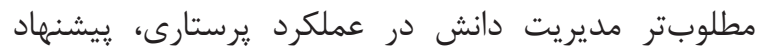

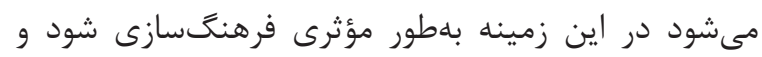

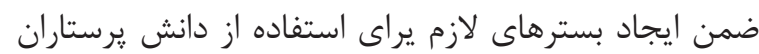

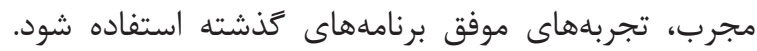

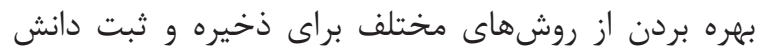

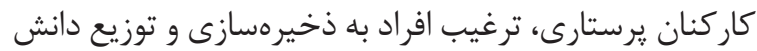

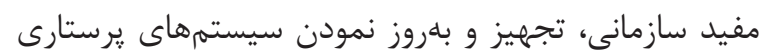

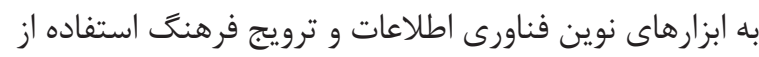

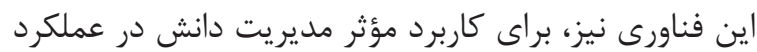

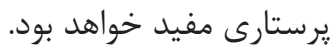

\section{سياسگزارى}

يزوهشكَران از معاونت يزوهشى دانشَاه و همكاران محترم

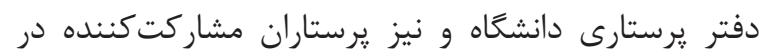

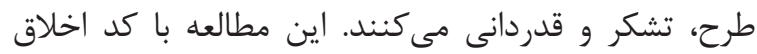

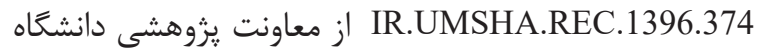
علوم يزشكى همدان به تصويب رسيده است. تضاد منافع در اين مطالعه تضاد منافع وجود ندارد.

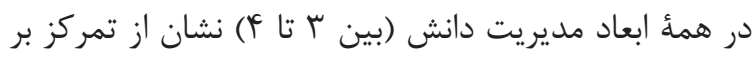

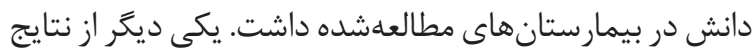

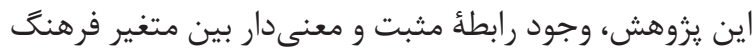

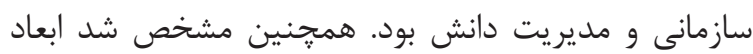

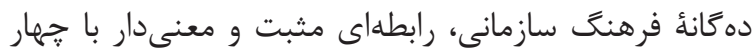

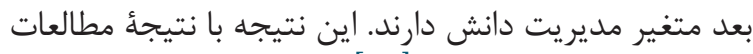
Ramezankhani

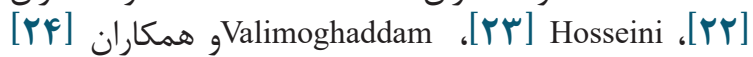

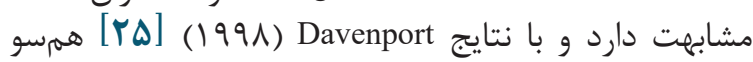

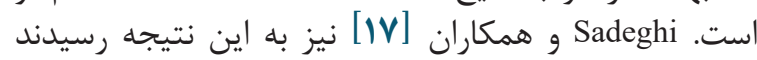

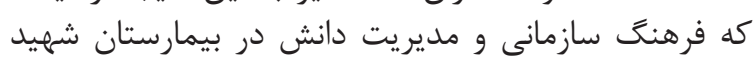

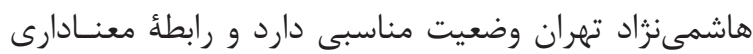

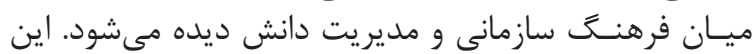

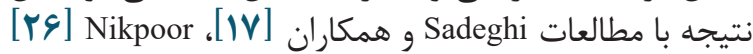

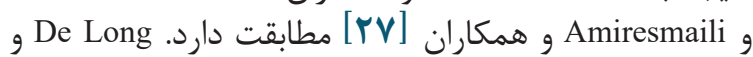

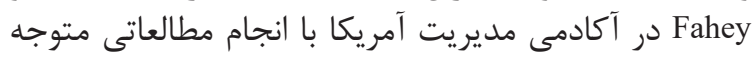

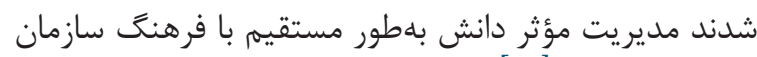

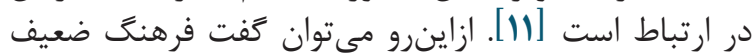

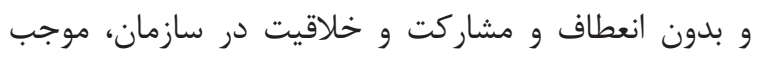

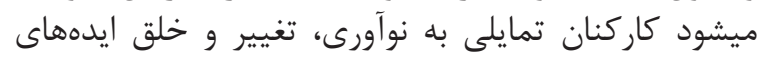

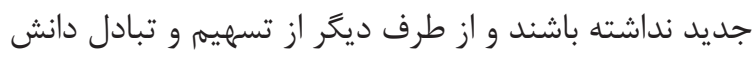

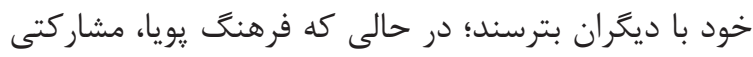

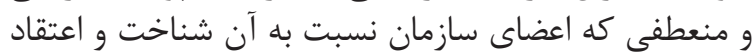

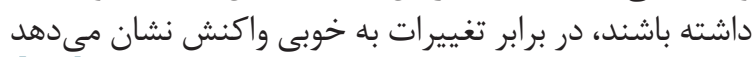

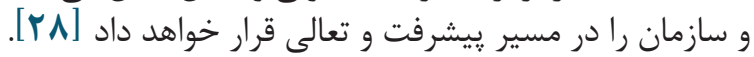

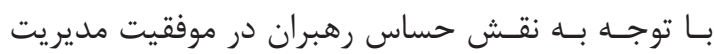

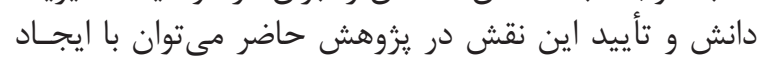

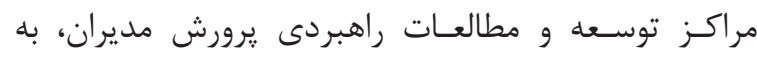

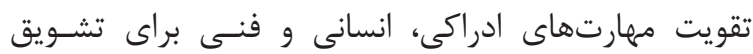

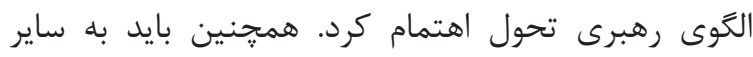
عوامل فرهنگ

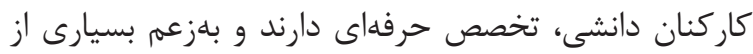

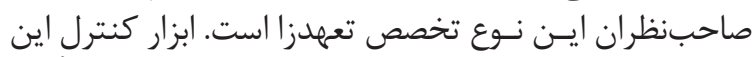

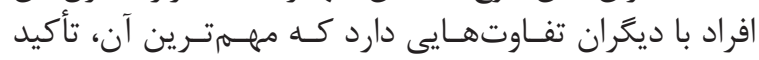

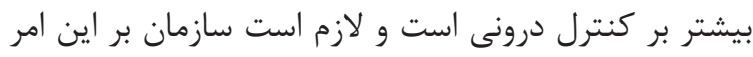

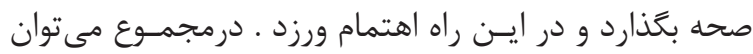

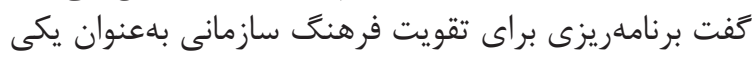

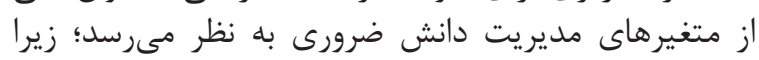

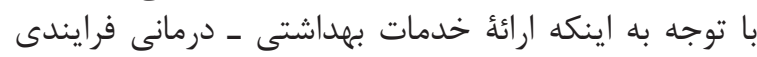

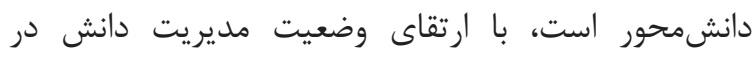

\section{References}

1. Hashemi S MMY, Mohamadi Moghadam E. Knowledge management, requirements. Journal of Human Resources management Development and Support. 2011;6(19):101- 30.

2. Fattahiyan S, Fattahiyan A, Omeidi K. The Relationship between Organizational Knowledge Strategies and Processes Knowledge Management. World of Science Journal. 2013;2:148-64.

3. Tseng F-Ch FY-J. Exploring the Influence of. Journal of Business Ethics. 2011;101:325-42. https://doi.org/10.1007/s10551-010-0725-5

4. Habibi F, Seyedi A, Bayat R, Haji Karimi A. A review of organizational empowerment with emphasis on knowledge management. Journal of Scientific Research and Development. 2015;2(4):229-32. 
5. Lim DH, Song JH, Yoon SW. Trends and issues in integrating knowledge management and organizational learning for workplace performance improvement. Handbook of human resource development. 2014:369-85.

6. Nonaka I, Takeuchi H. The knowledge creation company: how Japanese companies create the dynamics of innovation.

7. Sock W. Innovations of knowledge management: publisher of Innovation schooly and professional press; 2010.

8. Wang CL, Ahmed Perveiz K. Structure Dimensions for Knowledge Based Organizations. 2003;17(1):12. https://doi. org/10.1108/13683040310466726

9. Kooshkii M, Sepahvand R. The Relationship between Organizational Culture and the Use of ICT (Case Study: Maskan Bank Lorestan Province). Asian Journal of Research in Business Economics and Management. 2015;5(7):106-19.

10. Wang D, Su Z, Yang D. Organizational culture and knowledge creation capability. Journal of knowledge management. 2011 May 31;15(3):363-73.

11. De Long DW, Fahey L. Diagnosing cultural barriers to knowledge management. Academy of Management Executive. 2000;14:113.

12. Yew Wong K. Critical success factors for implementing knowledge management in small and medium enterprises. Industrial management \& Data systems. 2005;105(3):261-79.

13. Smith SN, Almirall D, Prenovost K, Goodrich DE, Abraham KM, Liebrecht C, Kilbourne AM. Organizational culture and climate as moderators of enhanced outreach for persons with serious mental illness: results from a cluster-randomized trial of adaptive implementation strategies. Implementation Science. 2018;13(1):93.

14. Mirghafori H, Farhang Nejad MA, Sadeghi Arani Z. Assessment the function of health services in Yasd province in application of knowledge management process. Journal of Health Administration. 2010;13(39):79-88.

15. Probst G, Stephen R, Romhardt K. Managing Knowledge: Building Blocks for Success. Canada: John Wiley \& Sons. 1999.

16. Perra BM. Leadership: the key to quality outcomes. Nurs Adm Q. 1992;24.

17. Sadeghi A, Jafari H, Khodayari R. The Relationship between Elements of Organizational Culture and Knowledge Management Case Study: HashemiNejad Hospital in Tehran Hospital. 2011; 10: 50-57.

18. Amiresmaeli MR, Nequeimoghaddam M, Khos- ravi S. Relationship between dimensions of organizational culture and knowledge management: The Case of Kerman University of Medical Sciences., Health Information Management.2014; 11: 770-778.

19. Tong C, Wah-Tak W, Wong A. The Impact of Knowledge Sharing on the Relationship between Organizational Culture and Job Satisfaction: the Perception of Information Communication and Technology (ICT) Practitioners in Hong Kong. International Journal of Human Resource Studies.2013;3:9-37.https://doi.org/10.5296/ijhrs. v3i1.3112

20. R Bose. Knowledge management metrics. 457-68, s.1. : Industrial Management \& Data Systems.2004; 104(6).https://doi. org/10.1108/02635570410543771

21. Ramezankhani A, Mahfoozpour S, Daneshkohan A, Danesh G. Comparison of correlation between knowledge management and organizational culture at public and private hospitals of Shiraz City. Journal Of Health Promotion Management. 2015;4:32.

22. Mirbelochezhi A, Movahedrad V, Bameri A, editors. The relationship between organizational culture and knowledge management in nursing Hospitals in the city of Iranshahr. Modern Research's in Managment,Economics and Accounting; 2015; Istanbul-Turkey.

23. Hosseini R. Survey the status of knowledge management in Ferdowsi University According to Nonaka and its relationship with corporate culture. Journal of Rahbord. 2005;9(59):191-216.

24. Valimoghaddam S, Tabibi S, Nasiripour A. Relationship between organizational culture and knowledge management in Zanjan teaching hospitals. Journal of Zanjan University of Medical Sciences. 2010;17(69):4-91.

25. Davenport T, Prusak L. Working knowledg:How organizations manage what they know. Boston: Harvard Business school press.; 1998.

26. Nikpoor A, Salajeghe DS. The relationship between knowledge management and organizational culture from the perspective of faculty members in Kerman. Farasoye Modiriat. 2010;14:18.

27. Amiresmaili MR, Nekooi Moghadam M, Khosravi S, Mirzaei S, Oroomiei N. The Relationship between Organizational Culture Components and Knowledge Management: Case Study of Kerman University of Medical Sciences. Health Inf Manage. 2015;11(6):778.

28. Arjmandi T. The relationship between organizational culture and preservation experts. 2007;24(1); 24(1): 102-24. 
29. Alvani M, Mohammadi S, Mirzaei M. The Effect of Organizational Culture on the Productivity of Human Resources. Journal of Industrial Strategic Management.2010. 9(28):25-38.

30. Haj Nasseri A, Javadi A. The Relationship between Organizational Culture and Performance Evaluation Systems in Tehran Stock Exchange Manufacturing Companies. Accounting Advances, 2013; 5 (2): 127-163.

31. Soltan HM, Mousavi Z. The survey of relative ratio of knowledge management on organizational health in Esfahan Physical Education Offices. 